\title{
Interaction between dexamethasone and butyrate in apoptosis induction: non-additive in thymocytes and synergistic in a $T$ cell-derived leukemia cell line
}

\author{
David Bernhard ${ }^{1}$, Markus Löffler², Bernd L. Hartmann², \\ Minoru Yoshida ${ }^{3}$, Reinhard Kofler ${ }^{2}$ and Adam Csordas ${ }^{\star 1}$ \\ 1 Institute of Medical Chemistry and Biochemistry, University of Innsbruck, \\ Fritz-Pregl-Str. 3, A-6020 Innsbruck, Austria \\ 2 Institute for General and Experimental Pathology, Division of Molecular \\ Pathophysiology, University of Innsbruck, Fritz-Pregl-Str. 3, A-6020 \\ Innsbruck, Austria \\ 3 Department of Biotechnology, Graduate School of Agriculture and Life \\ Sciences, The University of Tokyo, Yayoi 1-1-1, Bunkyo-ku, Japan \\ * corresponding author: Adam Csordas, Institute of Medical Chemistry and \\ Biochemistry, University of Innsbruck, Fritz-Pregl-Str. 3, A-6020 Innsbruck, \\ Austria. tel: +43 512507 3504; fax: +43 512507 2872; \\ e-mail: Adam.Csordas@uibk.ac.at
}

Received 13.07.98; revised 30.03.99; accepted 13.04.99 Edited by J. Cidlowski

\begin{abstract}
In thymocytes butyrate and trichostatin $A$ are unable to augment dexamethasone-induced apoptosis. In cultured rat thymocytes the extent of apoptosis induced by dexamethasone alone did not increase by addition of $0.1-10 \mathrm{mM}$ butyrate. Even more pronounced was the non-additive interrelationship between dexamethasone and trichostatin A, as trichostatin A-induced apoptosis was not only blocked by the presence of dexamethasone but dexamethasoneinduced apoptosis was also partially inhibited in the presence of $0.1-0.5 \mu \mathrm{M}$ trichostatin A. The fact that the non-additive relationship with dexamethasone for apoptosis induction was observed with both histone deacetylase inhibitors suggests that in thymocytes this phenomenon is related to histone acetylation. In contrast to this, in the human T cell-derived leukemia cell line $\mathrm{CEM}-\mathrm{C} 7 \mathrm{H} 2$, dexamethasone did not block butyrate- or trichostatin A-induced apoptosis; moreover, butyrate, in the concentration range of $0.1-1 \mathrm{mM}$, had a marked synergistic effect on dexamethasone-induced apoptosis. This synergism, however, was not mimicked by trichostatin $A$, indicating that the effect is not related to histone acetylation but rather due to a pleiotropic effect of butyrate. Furthermore, in CEM-C7H2 cells, at higher concentrations of butyrate $(5-10 \mathrm{mM})$ or trichostatin A (0.4$0.8 \mu \mathrm{M})$, there was a minor but reproducible antagonistic effect of dexamethasone on apoptosis induced by each of the two histone deacetylase inhibitors, suggesting that this antagonistic effect too, is related to histone hyperacetylation.
\end{abstract}

Keywords: apoptosis; butyrate; dexamethasone; thymocytes; CEM-C7H2; T cell-derived leukemia cells; short-chain fatty acids; trichostatin $\mathrm{A}$

\section{Introduction}

The short-chain fatty acid butyrate is, at millimolar concentrations, an inhibitor of histone deacetylases in animal cells. In cell cultures, butyrate induces a number of effects, such as reversible arrest of cell division, induction of differentiation and alterations in gene expression. ${ }^{1-3}$ Although the expression of certain genes is induced or upregulated, several genes remain unaffected and the transcription of yet other genes is even inhibited by butyrate (for reviews see ${ }^{2,3}$ ). In recent years, butyrate, a product of fermentation by gut bacteria, was found in vitro to be an inducer of apoptosis for transformed $^{4-8}$ as well as non-transformed intestinal epithelial cell lines. ${ }^{9}$ Subsequently it was shown that butyrate is an inducer of apoptosis in several other cell types too, such as Burkitt's lymphoma BL-30 cells, ${ }^{10}$ HL60 leukemia cells, ${ }^{11}$ HIV-1 transformed human cells, ${ }^{12}$ MCF-7 breast cancer cells, ${ }^{13}$ transformed lung fibroblasts ${ }^{14}$ and $\mathrm{Y}-79$ retinoblastoma cells. ${ }^{15}$

In spite of its ability to activate a number of genes, such as the 'dormant' fetal $\gamma$-globin gene, ${ }^{16}$ it was also noted that butyrate inhibits the transcriptional activation of several steroid hormone-dependent genes. An inhibitory effect of butyrate was shown for estrogen-mediated gene expression in the chicken oviduct, ${ }^{17}$ for glucocorticoiddependent genes in HTC cells, ${ }^{18,19}$ in cultured mammary gland fragments ${ }^{20,21}$ and for thyroid-mediated gene expression in rat pituitary tumor cells. ${ }^{22}$ Interestingly, in rat glioma C6 cells, butyrate was found to block the glucocorticoid-induced increase in glycerol phosphate dehydrogenase ${ }^{23}$ but had no effect on glucocorticoid induction of glutamine synthase activity; ${ }^{24}$ in HeLa cells the induction of alkaline phosphatase by dexamethasone was, however, potentiated by butyrate. ${ }^{25}$ The apparently contradictory effects of butyrate on steroid-mediated gene expression may be explained by a different role of chromatin structure for the regulation of individual genes. A number of studies point to an essential role of chromatin remodelling in the formation of the transcription initiation complex of glucocorticoid-sensitive genes by demonstrating that modulations of nucleosomal positioning and nucleosomal conformation in the promoter region are involved in the transactivation process. ${ }^{26-33}$ The fact that the glucocorticoid-dependent disruption of a specific nucleosome of the mouse mammary tumor virus is prevented by the presence of butyrate ${ }^{34}$ is consistent with the idea that ongoing histone deacetylation is a 
requirement for chromatin remodelling events. It appears plausible that rapid waves of acetylation and deacetylation are required for chromatin remodelling processes to take place, and these are blocked by deacetylase inhibitors. More recently, in agreement with this model, it was shown that low concentrations of butyrate or trichostatin A enhance basal as well as hormone-induced transcription from the MMTV-promoter, while higher concentrations of the histone deacetylase inhibitors reduce both these types of MMTV transcription. ${ }^{35}$ Thus, a moderate increase in histone acetylation activates the mouse mammary tumor virus promoter, presumably by helping to remodel a nucleosomal structure, whereas the remodelling process is prevented by a more efficient inhibition of histone deacetylation, ${ }^{35}$ i.e. by the establishment of the hyperacetylated state.

The triggering mechanism of the early events in glucocorticoid-induced apoptosis is poorly understood. We were therefore interested to test whether transactivation processes-in analogy to the above-mentioned model of the MMTV promoter-are operative in dexamethasoneinduced apoptosis. Thus, the question was raised whether and in what way the histone deacetylase inhibitors butyrate and trichostatin $A^{36,37}$ interact with dexamethasone-induced apoptosis.

Two different mechanisms have been discussed for glucocorticoid-induced apoptosis: first, transactivation according to the paradigmatic glucocorticoid-mediated gene activation model of the MMTV promoter, and second, repression, which could occur either by DNA binding of the receptor-ligand complex or via protein-protein interactions between the ligand-activated receptor and, for instance, the sequence-specific transcription factor AP-1. Furthermore, to get an idea whether the observed effects are cell typespecific, we tested the effects of butyrate on dexamethasone-induced apoptosis in two different types of $\mathrm{T}$ cells, namely in rat thymocytes and in the T-cell derived human leukemia cell line CEM-C7H2. ${ }^{38}$ Thus, the question was raised whether dexamethasone-induced apoptosis is stimulated by the additional presence of butyrate or, whether butyrate is antagonistic to dexamethasoneinduced apoptosis-in analogy to the above-mentioned examples of inhibition of glucocorticoid-mediated gene expression by butyrate.

In the present study we found that in thymocytes, butyrate-on its own an apoptosis inducer-did not increase the extent of dexamethasone-induced apoptosis and this effect was mimicked by trichostatin $A$, which in addition showed a partial inhibition of dexamethasoneinduced apoptosis. In contrast, in the leukemia cell line CEM-C7H2, dexamethasone did not prevent a dosedependent increase in the percentage of apoptotic cells by the additional presence of butyrate or trichostatin $A$, and at 0.1-1 mM butyrate a marked synergism between butyrate- and dexamethasone-induced apoptosis was observed. This effect, however, was not mimicked by trichostatin A. Furthermore, in the $\mathrm{CEM}-\mathrm{C} 7 \mathrm{H} 2$ cells too, at higher concentration ranges of butyrate and trichostatin $A$, respectively, an antagonistic interaction of dexamethasone with apoptosis induction was observed.

\section{Results}

\section{Butyrate-induced apoptosis in thymocytes}

The thymocytes were set up for primary cultures as described in Materials and Methods and suspended in RPMI 1640 medium. First the sensitivity of the ex vivo cultured thymocytes for butyrate-induced apoptosis was tested. Figure 1 shows the time- and dose-dependence of butyrateinduced apoptosis in ex vivo cultured thymocytes. Incubation for $15 \mathrm{~h}$ with $1 \mathrm{mM}$ of butyrate induced $15-20 \%$ apoptosis, with $5 \mathrm{mM}$ butyrate, $25-30 \%$ apoptosis. Within this time frame of $15 \mathrm{~h}$, even with $10 \mathrm{mM}$ of butyrate the percentage of apoptotic cells did not rise above $35 \%$. Figure 1 also shows that more than $9 \mathrm{~h}$ were needed until apoptosis became markedly manifest. The percentages of apoptosis in Figure 1 as in all other figures of the paper are based on measurements of propidium iodide fluorescence as described in Materials and Methods.

\section{Dexamethasone-induced apoptosis in thymocytes}

Figure 2 shows the time- and dose-dependence of dexamethasone-induced apoptosis in primary cultures of rat thymocytes. After an incubation period of $12 \mathrm{~h}$, there was $10-$ $20 \%$ apoptosis with $10 \mathrm{nM}$ of dexamethasone and by applying 100 and $1000 \mathrm{nM}$ of dexamethasone, the extent of apoptosis induced in $12 \mathrm{~h}$ increased to about 25 and $30 \%$, respectively. After $6 \mathrm{~h}$ of incubation, at all three concentrations of dexamethasone tested, the percentage of apoptotic cells was below $10 \%$. With $100 \mathrm{nM}$ of dexamethasone, the number of apoptotic cells reached more than $40 \%$ after $15 \mathrm{~h}$. Thus, in most of the thymocytes DNA loss as a result of chromatin fragmentation, which is measured in the propidium iodide fluorescence-assay, became manifest in the time period between 9 and $12 \mathrm{~h}$.

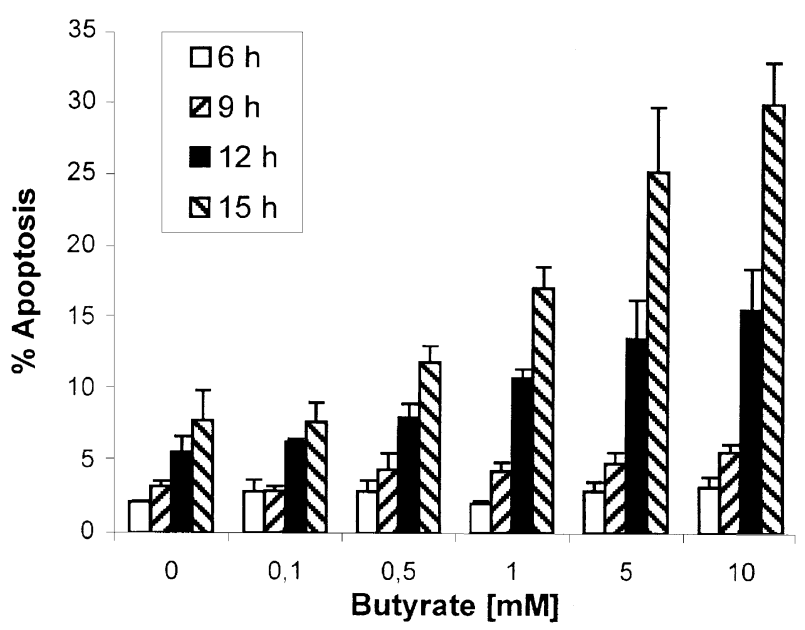

Figure 1 Concentration- and time-dependence of butyrate-induced apoptosis in ex vivo cultured rat thymocytes. As indicated in the insert, apoptosis was measured after incubation times of $6,9,12$ and $15 \mathrm{~h}$ with butyrate concentrations in the range of $0.1-10 \mathrm{mM}$ butyrate. The data are means of triplicates \pm S.D. of a representative experiment 


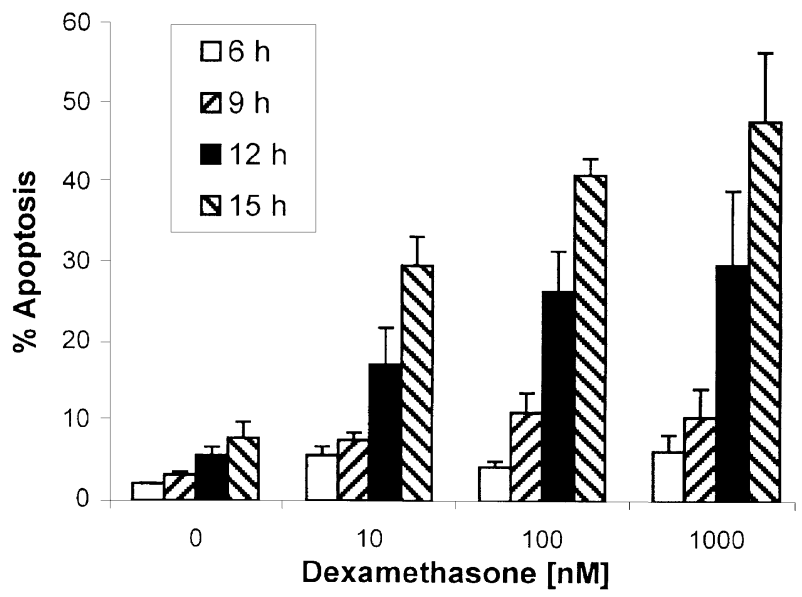

Figure 2 Concentration- and time-dependence of dexamethasone-induced apoptosis in ex vivo cultured rat thymocytes. Apoptosis was assayed at the concentrations of 10,100 and $1000 \mathrm{nM}$ of dexamethasone and after the incubation times of $6,9,12$ and $15 \mathrm{~h}$, respectively. The data are means of triplicates \pm S.D. of a representative experiment

\section{Butyrate does not increase the extent of apoptosis induced by dexamethasone alone in thymocytes}

In several earlier studies butyrate was reported to be an inhibitor of glucocorticoid-induced gene expression; ${ }^{17-22,34}$ on the other hand, for the transcriptional activation of the MMTV promoter, butyrate (and trichostatin A) exhibited a biphasic effect, being an activator at lower concentrations and an inhibitor above a certain concentration. Against this background, it was of interest to test whether any analogy could be detected in the impact of butyrate on glucocorticoidinduced apoptosis.

Figure 3 shows the effects of butyrate at the concentration range $0.1-10 \mathrm{mM}$ on dexamethasone-induced apoptosis in primary cultures of rat thymocytes at the dexamethasone concentration of $100 \mathrm{nM}$ and after an incubation time of $15 \mathrm{~h}$.

Butyrate by itself is an apoptosis inducer in a dosedependent manner (Figures 1 and 3); in the concentration range of $0.1-10 \mathrm{mM} \mathrm{Na-butyrate,} \mathrm{there} \mathrm{was} \mathrm{a} \mathrm{significant}$ increase $(P=0.01)$ in the percentage of apoptosis induced by butyrate alone, starting at less than $10 \%$ and increasing to about $30 \%$. Within the same time period, $100 \mathrm{nM}$ dexamethasone applied alone induced about 40\% apoptosis. As shown in Figure 3, when in addition to $100 \mathrm{nM}$ dexamethasone various concentrations of butyrate were applied in the range of $0.1-10 \mathrm{mM}$, no significant increase in the percentage of apoptosis could be observed. In spite of the additional presence of apoptosis-inducing concentations of butyrate, the percentage of apoptotic cells remained unchanged, namely $40-50 \%$ as induced by $100 \mathrm{nM}$ dexamethasone alone. The differences in the percentages of apoptotic cells at the various concentrations of butyrate under combined application of the two apoptosis inducers were without statistical significance $(P=0.05)$. Although clearly a non-additive relationship between the two apoptosis inducers was demonstrated, it is not possible to

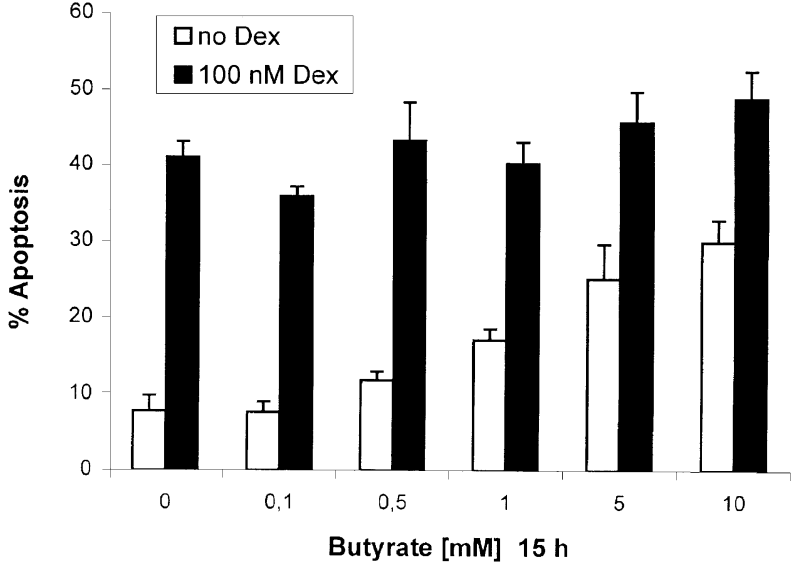

Figure 3 Non-additive interaction between dexamethasone-induced and butyrate-induced apoptosis in thymocytes. Apoptosis was measured in the presence of $100 \mathrm{nM}$ dexamethasone (Dex) and the indicated butyrate concentrations, respectively, after an incubation time of $15 \mathrm{~h}$. Note the concentration-dependent increase in the percentage of apoptosis with the increasing concentrations of butyrate alone which was statistically significant $(P=0.01)$ in the concentration range tested $(0.1-10 \mathrm{mM}$ butyrate) and in contrast to this, a lack of increase in the percentage of apoptotic cells under combined application of the two apoptosis inducers; the differences in the percentages of apoptosis at the various concentrations of butyrate under combined application of the two drugs were without statistical significance even at the level $P=0.05$

draw any conclusions as to which of the two apoptosis inducers inhibits the other or whether they mutually inhibit each other and if so to what extent. The unchanged percentage of apoptosis induced by $100 \mathrm{nM}$ dexamethasone alone, however, suggests that it is dexamethasoneinduced apoptosis which causes a blocking of butyrateinduced apoptosis, as various concentrations of butyrate are unable to increase the extent of apoptosis induced by dexamethasone alone. Since butyrate is an inhibitor of histone deacetylases, in the following it was tested whether trichostatin A, a structurally unrelated histone deacetylase inhibitor, also shows a similar type of non-additive relationship with dexamethasone for apoptosis induction.

\section{Trichostatin A does not increase the extent of apoptosis induced by dexamethasone alone in thymocytes}

Figure 4 shows the effects of various concentrations of trichostatin A on apoptosis induced by $100 \mathrm{nM}$ dexamethasone after an incubation period of $12 \mathrm{~h}$ in primary cultures of rat thymocytes. When trichostatin $\mathrm{A}(0.01-5 \mu \mathrm{M})$ was applied alone, it showed a significant dose-dependent increase of apoptosis. Dexamethasone alone induced in $12 \mathrm{~h}$ about $25 \%$ of apoptosis (as also shown in Figure 2). The additional presence of $0.01-5 \mu \mathrm{M}$ trichostatin $\mathrm{A}$ was not able to increase the extent of apoptosis induced by $100 \mathrm{nM}$ dexamethasone alone but led at $0.1-0.5 \mathrm{mM}$ to a significant partial inhibition of dexamethasone-induced apoptosis. The fact that both butyrate (Figure 3) and trichostatin A (Figure 4) exhibited a non-additive interaction with dexamethasone in inducing apoptosis in thymocytes strongly suggests that the 


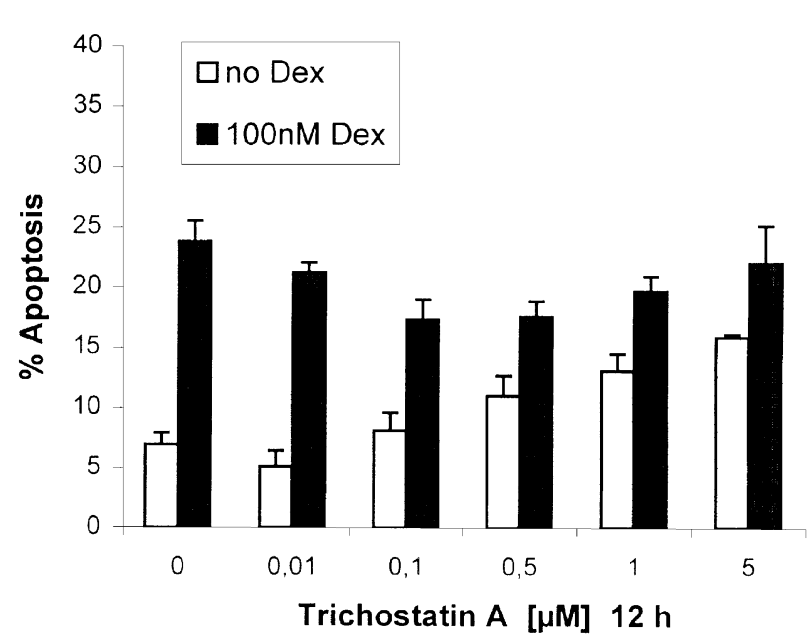

Figure 4 Non-additive interaction of dexamethasone-induced with trichostatin A-induced apoptosis in thymocytes. Apoptosis was measured with $100 \mathrm{nM}$ dexamethasone (Dex) and the indicated concentrations of trichostatin $A$ $(0.01-5 \mu \mathrm{M})$, respectively, after an incubation time of $12 \mathrm{~h}$. There was a significant dose-dependent increase in the extent of apoptosis with trichostatin A alone $(P=0.001)$ in the range of concentrations tested $(0.01-5 \mu \mathrm{M})$; however, there was no increase of apoptosis induced by dexamethasone when various concentrations of trichostatin A were added; at the concentrations 0.1 and $0.5 \mu \mathrm{M}$ there was even a statistically significant $(P=0.01)$ inhibition of dexamethasone-induced apoptosis under combined application of the two drugs

acetylation status of histones, even if only in a very limited region of chromatin, is in some way related to this phenomenon.

\section{Butyrate-induced apoptosis in CCRF-CEM leukemia cells}

Next we investigated the effect of butyrate on dexamethasoneinduced apoptosis in the T cell-derived human leukemia cell line $\mathrm{CEM}-\mathrm{C} 7 \mathrm{H} 2$ which has been used as a model in several previous investigations on the mechanism of glucocorticoidinduced apoptosis. ${ }^{39-42}$ As early as 1982 it was reported that butyrate is cytotoxic to CCRF-CEM cells; ${ }^{43}$ however, whether cell death occurred through apoptosis, and further, a possible impact of butyrate on glucocorticoid-induced cell death was not investigated. To address these issues, we first tested the sensitivity of the $\mathrm{CEM}-\mathrm{C} 7 \mathrm{H} 2$ line to butyrate. Figure 5 shows that in $\mathrm{CEM}-\mathrm{C} 7 \mathrm{H} 2$ cells apoptosis at the concentration range of 5-10 mM butyrate was clearly detectable after $24 \mathrm{~h}(20-$ $40 \%$ ) and reached a maximum of $80 \%$ after $48 \mathrm{~h}$.

\section{Dexamethasone-induced apoptosis in CCRF-CEM leukemia cells}

Likewise, dexamethasone-induced apoptosis was analyzed in a broad concentration range and at time intervals of 24 and $48 \mathrm{~h}$ of incubation with the drug. As shown in Figure 6, incubation with $100 \mathrm{nM}$ of dexamethasone resulted in 60$80 \%$ apoptosis after $48 \mathrm{~h}$ and about $10 \%$ after $24 \mathrm{~h}$ (in addition to spontaneous apoptosis). Increasing the dexamethasone concentration by an order of magnitude to $1000 \mathrm{nM}$ did not seem to change much the time course of apoptosis.

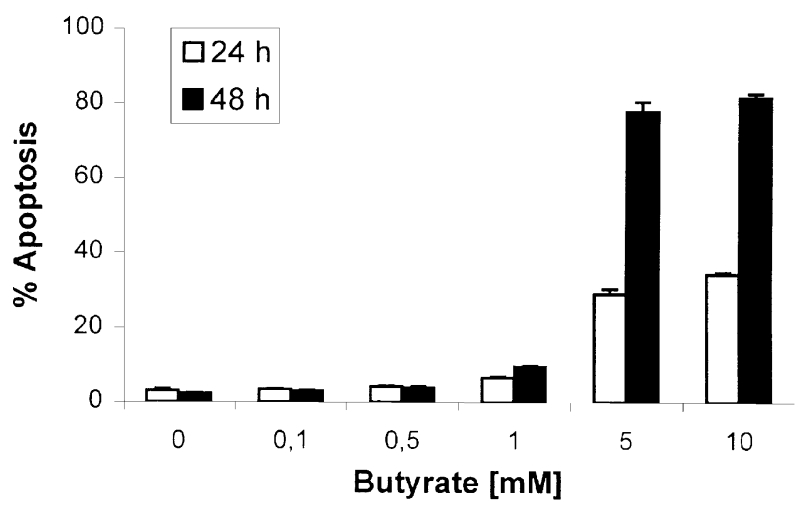

Figure 5 Concentration- and time-dependence of butyrate-induced apoptosis in $\mathrm{CEM}-\mathrm{C} 7 \mathrm{H} 2$ cells. Data are means of triplicates \pm S.D. of a representative experiment

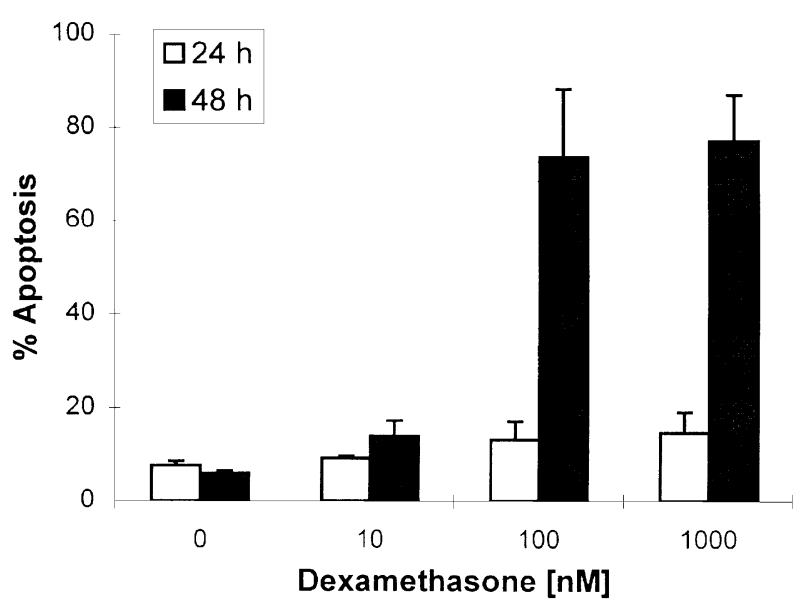

Figure 6 Concentration- and time-dependence of dexamethasone-induced apoptosis in $\mathrm{CEM}-\mathrm{C} 7 \mathrm{H} 2$ cells. Apoptosis was assayed at three different concentrations of dexamethasone (Dex) $(10,100$ and $1000 \mathrm{nM})$ after 24 and $48 \mathrm{~h}$ of incubation. Data are means \pm S.D. of triplicates of a representative experiment

\section{Dexamethasone does not block butyrate-induced apoptosis in CCRF-CEM leukemia cells}

Figure 7 shows the percentages of apoptosis induced in CEM$\mathrm{C} 7 \mathrm{H} 2$ leukemia cells by $1000 \mathrm{nM}$ dexamethasone, various concentrations of butyrate and under combined application of the two drugs, respectively, after the incubation time of $36 \mathrm{~h}$. The percentage of spontaneous apoptosis in the absence of both drugs, and further, the percentages of dexamethasoneinduced apoptosis (1000 nM) - in the absence of butyrateare shown at the zero butyrate value of the abscissa of the diagram in Figure 7. Spontaneous apoptosis was less than $5 \%$ and in the presence of $1000 \mathrm{nM}$ dexamethasone there was a further $10 \%$ apoptosis in $36 \mathrm{~h}$.

Figure 7 shows that the additional presence of butyrate led to a dose-dependent increase in the percentage of apoptotic cells. Moreover, at the concentrations of $0.1,0.5$ and $1 \mathrm{mM}$ there was a marked synergistic effect of butyrate on dexamethasone-induced apoptosis. When, 0.5 or $1 \mathrm{mM}$ 
butyrate was present in addition to dexamethasone, there was an approximately three- (about $30 \%$ instead of $10 \%$ ) and fourfold increase (about $40 \%$ instead of $10 \%$ ), respectively, in the percentage of apoptotic cells compared to that induced by $1000 \mathrm{nM}$ dexamethasone alone. It should be pointed out that $0.1,0.5$ and $1 \mathrm{mM}$ are concentrations of butyrate which are at the limit of the apoptosis-inducing ability and induce only a marginal extent of apoptosis (Figures 5 and 7), so the observed synergism is clearly an effect for which dexamethasone is a prerequisite. At the concentrations of 5 and $10 \mathrm{mM}$, however, a minor antagonistic effect between the two apoptosis inducers could be observed, as butyrate alone at these concentrations was a slightly more efficient apoptosis inducer than together with dexamethasone. This antagonistic effect was statistically not significant within the triplicates of one experiment but it was reproducibly present in all the experiments. The biphasic interaction of butyrate with dexamethasone-induced apoptosis was also observed in experiments with 10 and $100 \mathrm{nM}$ dexamethasone, respectively, as also the synergism in the concentration range $0.1-$ $1 \mathrm{mM}$ butyrate (data not shown); however, the extent of synergism was most pronounced at the highest dexamethasone concentration tested (1000 nM).

Again the question was raised whether the observed effects of butyrate were related to histone acetylation and therefore in the following the effects of trichostatin $A$ on dexamethasone-induced apoptosis were tested.

\section{Trichostatin A does not mimick the synergistic effect of butyrate on dexamethasone-induced apoptosis in CCRF-CEM leukemia cells}

Figure 8 shows the extent of apoptosis after 24 h of incubation induced by various concentrations of trichostatin A alone, and in the additional presence of $100 \mathrm{nM}$ dexamethasone. With

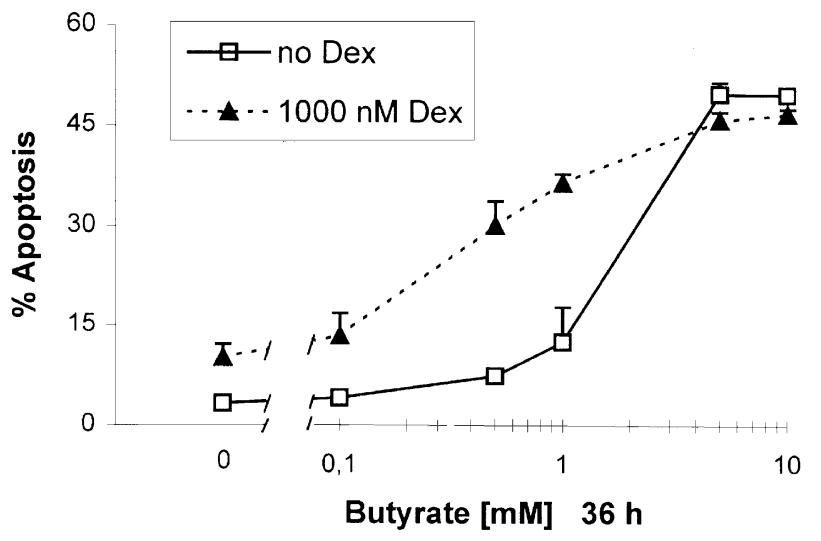

Figure 7 Stimulation of dexamethasone-induced apoptosis by butyrate in CEM-C7H2 leukemia cells. Apoptosis was measured $36 \mathrm{~h}$ after application of the apoptosis inducers. Butyrate exerts over the concentration range of $0.1-$ $1 \mathrm{mM}$ a significant $(P=0.001)$ dose-dependent increase of apoptosis induced by dexamethasone alone with synergism at the concentrations $0.1,0.5$ and $1 \mathrm{mM}$ butyrate $(P=0.001)$. Note the transition of the synergistic effect to an antagonistic one at 5 and $10 \mathrm{mM}$ butyrate; at these concentrations in the additional presence of $1000 \mathrm{mM}$ dexamethasone the percentage of apoptosis was lower than that induced by butyrate alone; although within the triplicates of an experiment this anatagonistic effect was at $P=0.05$ without statistical significance, it was observed in every experiment
$100 \mathrm{nM}$ dexamethasone alone there was about $10 \%$ apoptosis. This low percentage of dexamethasone-induced apoptosis was selected for testing the effect of trichostatin A, because in previous experiments performed at higher percentages of dexamethasone-induced apoptosis (data not shown), no inhibition of dexamethasone-induced apoptosis by trichostatin A was observed. Therefore, we tested whether the stimulating effect and/or the synergistic effect of butyrate on dexamethasone-induced apoptosis in CEM-C7H2 cells can be mimicked with trichostatin $A$. Figure 8 shows that dexamethasone does not block additional apoptosis induced by trichostatin A which clearly increases apoptosis in a dosedependent manner-and this is in analogy to butyrate. However, in contrast to the behavior of butyrate, no synergistic effect of trichostatin $A$ on dexamethasoneinduced apoptosis could be detected. The synergistic effects of butyrate on dexamethasone-induced apoptosis were observed at a lower concentration range of butyrate, at the limit of the apoptosis-inducing concentrations. However, no stimulating effect on dexamethasone-induced apoptosis was detected at 0.05 and $0.1 \mu \mathrm{M}$ trichostatin A. At concentrations above $0.1 \mu \mathrm{M}$, trichostatin $\mathrm{A}$ alone was a powerful apoptosis inducer. Again, as also observed at higher butyrate concentrations, trichostatin A-induced apoptosis at 0.4 and $0.8 \mu \mathrm{M}$ was slightly inhibited by dexamethasone $(100 \mathrm{mM})$. This antagonistic effect was statistically significant within the triplicates of an experiment only at $0.8 \mu \mathrm{M}$ but was reproducibly observed at 0.4 and $0.8 \mu \mathrm{M}$ in all experiments performed. Since the synergistic effect of butyrate on dexamethasone-induced apoptosis in $\mathrm{CEM}-\mathrm{C} 7 \mathrm{H} 2$ cells (Figure 7) was not mimicked by trichostatin $A$, we have to

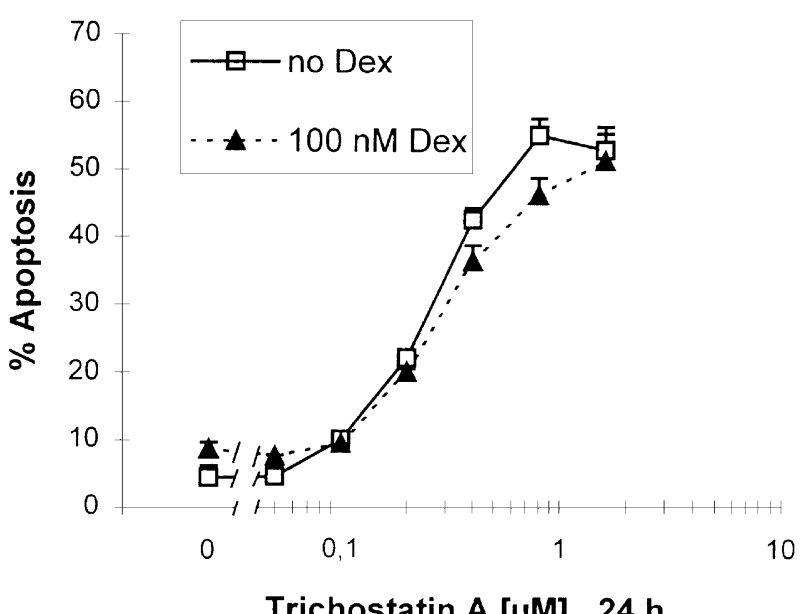

Figure 8 Lack of synergism in the extent of apoptosis under the combined application of trichostatin A and dexamethasone (Dex) in CEM-C7H2 leukemia cells. Apoptosis was measured $24 \mathrm{~h}$ after application of $100 \mathrm{nM}$ Dex and the indicated concentrations of trichostatin $A$. The combined application of the two apoptosis inducers did not result $(P=0.05)$ in any significant increase in the percentage of apoptosis when compared to that induced by trichostatin $A$ alone. At 0.4 and $0.8 \mu \mathrm{M}$ trichostatin $\mathrm{A}$ the percentage of apoptosis was lower under combined application with $100 \mathrm{mM}$ dexamethasone compared to that induced by trichostatin $\mathrm{A}$ alone; the difference at $0.8 \mu \mathrm{M}$ was significant $(P=0.001)$ but at both concentrations 0.4 and $0.8 \mu \mathrm{M}$ trichostatin $\mathrm{A}$ the effect was observed in each experiment even if the difference was not significant within the triplicates of one experiment 
conclude that this effect is not related to histone acetylation but is due to a pleiotropic effect of butyrate.

\section{Discussion}

The purpose of the present investigation was to examine whether and in what way the two histone deacetylase inhibitors butyrate and trichostatin $A$ exert an influence on dexamethasone-induced apoptosis, i.e. whether they inhibit or potentiate this apoptosis pathway. The aim of our study was also to test with the use of the two histone deacetylase inhibitors whether any analogy can be found between glucocorticoid-induced apoptosis and the models of glucocorticoid-mediated gene expression. Two different $T$ celltypes, namely thymocytes and the $T$ cell-derived leukemia cell line CEM-C7H2, were examined in order to get an idea of celltype specificity and cancero-selectivity of the observed effects.

Millimolar concentrations of butyrate cause hyperacetylation of histones (for reviews see ${ }^{44-47}$ ) and reversible arrest of the cell cycle in animal cell cultures, generally. In certain cell lines, a small proportion of the cells or most of them progress further and undergo cell death by apoptosis. Thus, butyrateinduced apoptosis shows a pronounced cell-type specificity, while reversible inhibition of cell growth, induction of differentiation markers and modulation of gene expression represent effects of butyrate (and trichostatin $A$ ) which were found in all animal cells investigated. As far as these latter three, general effects of butyrate are concerned, they are supposed to result from histone hyperacetylation, because they can also be induced by trichostatin $A$, an inhibitor of histone deacetylases structurally unrelated to butyrate which exerts its inhibiting effect already at micromolar concentrations. ${ }^{36,37}$ As shown in the present study, apoptosis was induced in the investigated $\mathrm{T}$ cells by butyrate at millimolar (Figures 1, 3, 5 and 7) and by trichostatin A at micromolar concentrations (Figures 4 and 8 ) which are the histone deacetylase inhibiting concentrations, respectively, of the two drugs. Thus, histone hyperacetylation appears to be a phenomenon which is coupled with apoptosis induced by butyrate or trichostatin $\mathrm{A}$. Consistent with a role of histone acetylation in apoptosis induction by short-chain fatty acids is also our observation (data not shown) that propionate and valerate, which are weaker inhibitors of histone deacetylases than butyrate, also functioned as apoptosis inducers in the investigated $\mathrm{CEM}-\mathrm{C} 7 \mathrm{H} 2$ cells but at higher concentrations than butyrate, i.e., at their deacetylase-inhibiting concentrations. Moreover, in a previous investigation with the colon adenocarcinoma cell line VACO5 it was reported that the apoptosis inducing efficiency of short-chain fatty acids correlated with their histone deacetylase-inhibiting ability. ${ }^{48}$ Global histone hyperacetylation per se, however, cannot be sufficient for triggering apoptosis since it is achieved by butyrate treatment in many animal cell types that do not undergo butyrate-induced apoptosis.

Recent studies clearly demonstrate that butyrate-induced apoptosis is strongly cell type-specific. For instance, butyrate caused reversible arrest in the G1 and G2 phase of untransformed rat fibroblasts but was cytotoxic to the SV40 transformed derivation of the cell line. ${ }^{49}$ In a study with colon cancer cells, some of the investigated adenoma cell lines were more sensitive to butyrate-induced apoptosis than the carcinoma cell lines. ${ }^{6}$

Glucocorticoids induce apoptosis with a high degree of specificity in certain lymphoid cells, and it is not clear why other cell types which also possess glucocorticoid receptors are not sensitive. ${ }^{50}$ Perhaps in the insensitive cell types the relevant chromatin region is not appropriately prepared for receiving the activated glucocorticoid receptor.

It was recently reported that the degree of histone acetylation is changing as a result of dexamethasoneinduced apoptosis in thymocytes. ${ }^{51}$ The two $\mathrm{T}$ cell types investigated in the present study proved to be sensitive to apoptosis induction by dexamethasone as well as butyrate (or trichostatin A). It remains to be seen whether dexamethasone-sensitivity is always coupled with sensitivity to butyrate- (and trichostatin A)-induced apoptosis.

Whereas a transactivation mechanism was suggested for dexamethasone-induced apoptosis in thymocytes, ${ }^{52}$ in human leukemia cells, a repressive mechanism was favored. ${ }^{53}$ The different effects of butyrate on dexamethasone-induced apoptosis in thymocytes and in $\mathrm{CEM}-\mathrm{C} 7 \mathrm{H} 2$ leukemia cells observed in our study are certainly consistent with the concept that the mechanisms of dexamethasone-induced apoptosis in these two $\mathrm{T}$ celltypes are not identical. However, the effects of the two histone deacetylase-inhibitors suggest that histone acetylation may be the common denominator playing a role in dexamethasone-induced as well as in butyrate-induced (and trichostatin A-induced) apoptosis.

The results of the present investigation are consistent with the interpretation that in thymus as well as in CEM$\mathrm{C} 7 \mathrm{H} 2$ cells, dexamethasone causes analogous structural changes in chromatin structure for apoptosis induction as butyrate (or trichostatin A). One may speculate that in thymus a gene becomes fully turned on by dexamethasone, so that the presence of butyrate (or trichostatin A) cannot lead to any further activation. If, however, in the leukemia cell line $\mathrm{CEM}-\mathrm{C} 7 \mathrm{H} 2$ (regardless whether the mechanism is glucocorticoid-mediated activation or repression), in a relevant promoter region a repressing chromatin structure is not fully lifted by dexamethasone, then butyrate (or trichostatin A) would cause, in a dose-dependent manner, a more efficient removal of this repressing chromatin structure and thus lead to an increased extent of apoptosis. This would explain the dose-dependent increase of butyrate- (and also trichostatin A)-induced apoptosis in the presence of dexamethasone in CEM$\mathrm{C} 7 \mathrm{H} 2$ cells. The antagonistic effect on apoptosis at higher concentrations of the histone deacetyase inhibitors in CEM$\mathrm{C} 7 \mathrm{H} 2$ cells could be explained by a process which would become operative only after a more accessible chromatin structure has been established. That such changes in chromatin structure require some time till a certain level of hyperacetylation is reached, may explain the modest extent of this anti-apoptotic effect and that it was observed only at certain higher concentrations of the histone deacetylase inhibitors. According to this model, dexamethasone and butyrate (and trichostatin A) would target the same genes for apoptosis induction and butyrate-induced apoptosis 
would function as a short-cut of dexamethasone-induced apoptosis. If so, dexamethasone-sensitive cells should also be butyrate-sensitive. The model, however, does not demand that in turn, butyrate-sensitive cells have also to be glucocorticoid-sensitive. This is certainly not the case and it is quite conceivable that butyrate, by the changes it causes in chromatin structure, may activate apoptosispromoting genes which are not targets of the activated glucocorticoid receptor. Further studies should clarify whether the 'short-cut by butyrate' can be generalized for other glucocorticoid-sensitive cell types. It should be emphasized in this context that there is experimental evidence for a short-cut by histone deacetylase inhibitors -in the absence of the hormone-for the activation of the otherwise hormone-dependent MMTV promoter.

In the CEM-C7H2 leukemia cell line, at a lower concentration range of butyrate, a pronounced synergistic effect was observed on dexamethasone-induced apoptosis. Since this effect, however, was not mimicked by trichostatin $A$, it cannot be related to histone acetylation but rather to a pleiotropic effect of butyrate.

Butyrate was reported to have pleiotropic effects on the cytoskeleton, on mitochondria and on the cell membrane. ${ }^{48}$ It remains to be clarified in further studies which one, if any, of these effects participates in the above phenomenon. It should be possible to sort out-based on the hierarchy of various short-chain fatty acids in the efficiency of inducing a given pleioptropic effect-whether the synergism is mediated via mitochondria, the cytoskeleton or the plasma membrane. ${ }^{48}$ The differential sensitivity of nontransformed $T$ lymphocytes and the $T$ cell-derived leukemia cell line to the impact of butyrate on glucocorticoid-induced apoptosis has its implications for a higher cancero-selectivity in tumor therapy.

\section{Materials and Methods}

\section{Chemicals}

Butyric acid was from Fluka Chemie AG, Buchs, Switzerland. A stock solution of $2 \mathrm{M}$ sodium butyrate was prepared and the $\mathrm{pH}$ adjusted to 7.3 by addition of sodium hydroxide. (R)-Trichostatin A was purified from culture broths of Streptomyces hygroscopicus Y-50. RPMI 1640, fetal calf serum, glutamine, penicillin and streptomycin were purchased from Gibco BRL, Vienna, Austria; dexamethasone and propidum iodide were from Sigma, Vienna, Austria.

\section{Cells and cell culture}

$\mathrm{CEM}-\mathrm{C} 7 \mathrm{H} 2{ }^{38}$ a highly glucocorticoid-sensitive subclone of the acute lymphoblastic leukemia line CCRF-CEM-C7 $7^{54}$ was grown in $5 \% \mathrm{CO}_{2}$, saturated humidity, at $37^{\circ} \mathrm{C}$ in RPMI 1640 supplemented with $10 \%$ bovine calf serum (HyClone, Logan, UT, USA), $100 \mathrm{U} / \mathrm{ml}$ penicillin, $100 \mu \mathrm{g} / \mathrm{ml}$ streptomycin, and $2 \mathrm{mM}$ L-glutamine (Sigma, Vienna, Austria).

\section{Rat thymus culture}

Rat thymocytes were prepared from thymuses of 6-week-old female Sprague-Dawley rats. After sacrificing the animals by cervical dislocation, the thymuses were removed, placed in ice-cold RPMI
1640 medium and passaged through 100- and then through 200-mesh stainless steel nets. Cell density was adjusted to $1.5 \times 10^{6} / \mathrm{ml}$ and the cells were used immediately for the apoptosis experiments.

\section{Apoptosis assays}

Detection and quantification of apoptosis was performed by FACS analysis using two independent principles as apoptosis criteria, namely, nuclear staining with propidium iodide, on the one hand, and forward/sideward light scattering analysis, on the other. ${ }^{55}$ For the apoptosis assay $1 \times 10^{6}$ cells were centrifuged $\left(7 \mathrm{~min}, 4^{\circ} \mathrm{C}, 300 \times \mathrm{g}\right)$, the supernatant discarded and the pellet stained with $750 \mu \mathrm{l}$ freshly prepared propidium iodide $(50 \mu \mathrm{g} / \mathrm{ml}$ in $0.1 \%$ Triton $\mathrm{X}-100 / 0.1 \%$ sodium citrate solution). Subsequently, the nuclei were subjected to apoptosis analysis in a FACScan (Becton Dickinson, San Jose, CA, USA) using both fluorescence intensity of propidium iodide and forward/sideward light scattering as parameters for the determination of apoptosis. Based on propidium iodide fluorescence, nuclei in the sub-G1 marker window were considered to represent apoptotic cells. According to the light scattering analysis, apoptotic nuclei are recognized as being smaller (lower forward scatter values) and more granulated (higher sideward scatter values). Cell debris and small particles were excluded from the analysis based on forward/sideward scatter criteria as described. ${ }^{56}$ In addition, with butyrate as well as dexamethasone for both cell types, the appearance of a nucleosome ladder was demonstrated as a criterion for apoptosis (data not shown). However, since the analysis of a nucleosome ladder is not a suitable technique for quantification of the extent of apoptosis, and in view of the large number of the experimental conditions, the above-described two parameters of flow cytometric analyses were chosen as apoptosis criteria in the present study. In all the figures of the present study the percentages of apoptosis are calculated from the data of propidium fluorescence intensity.

\section{Statistical analysis}

Data are shown as mean values in all diagrams and are given as means of triplicates \pm S.D. of a representative experiment. Statistical significance was calculated using ANOVA and multiple Student's $t$-test comparisons.

\section{Acknowledgements}

This work was supported by grants from the Austrian Science Fund (SFBF002 and P11964) and the University of Innsbruck. We thank Ines Jaklitsch and Susanne Lobenwein for excellent technical assistance.

\section{References}

1. Kruh J (1982) Effects of sodium butyrate, a new pharmacological agent, on cells in culture. Mol. Cell. Biochem. 42: 65-82

2. Csordas A (1995) Toxicology of butyrate and short-chain fatty acids. In Role of Gut Bacteria in Human Toxicology and Pharmacology, Hill MJ, ed (London: Taylor and Francis) pp.105-127

3. Kruh J, Defer N and Tichonicky L (1995) Effects of butyrate on cell proliferation and gene expression. In Physiology and Clinical Aspects of Short-Chain Fatty Acids, Cummings $\mathrm{JH}$, Rombeau $\mathrm{JL}$ and Sakata T, eds (Cambridge University Press) pp. 275-288

4. Hague A, Manning AM, Hanlon KA, Huschtscha LI, Hart D and Paraskeva C (1993) Sodium butyrate induces apoptosis in human colonic tumour cell lines in a p53-independent pathway: implications for the possible role of dietary fibre in the prevention of large-bowel cancer. Int. J. Cancer 55: 498-505 
5. Heerdt BG, Houston MA and Augenlicht LH (1994) Potentiation by specific shortchain fatty acids of differentiation and apoptosis in human colonic carcinoma cell lines. Cancer Res. 54: 3288-3294

6. Hague A, Elder DJE, Hicks DJ and Paraskeva C (1995) Apoptosis in colorectal tumour cells: induction by the short chain fatty acids butyrate, propionate and acetate and by the bile salt deoxycholate. Int. J. Cancer 60: 400-406

7. Hague A and Paraskeva C (1995) The short-chain fatty acid butyrate induces apoptosis in colorectal tumour cell lines. Eur. J. Cancer Prev. 4: 359-364

8. McBain JA, Eastman A, Simmons DL, Petit GR and Mueller GC (1996) Phorbol ester augments butyrate-induced apoptosis of colon cancer cells. Int. J. Cancer 67: $715-723$

9. Tsujii M and DuBois RN (1995) Alterations in cellular adhesion and apoptosis in epithelial cells overexpressing prostaglandin endoperoxide synthase II. Cell 83: 493-501

10. Filippovich I, Sorokina N, Khanna KK and Lavin MF (1994) Butyrate-induced apoptosis in lymphoid cells preceded by transient over-expression of HSP70 mRNA. Biochem. Biophys. Res. Commun. 198: 257-265

11. Solary E, Bertrand R and Pommier Y (1994) Apoptosis of human leukemic HL-60 cells induced to differentiate by phorbol ester treatment. Leukemia 8: 792-797

12. Sadaie MR and Hager GL (1994) Induction of developmentally programmed cell death and activation of HIV by sodium butyrate. Virology 202: $513-518$

13. Mandal M and Kumar R (1996) Bcl-2 expression regulates sodium butyrateinduced apoptosis in human MCF-7 breast cancer cells. Cell Growth Differ. 7 311-318

14. Thomas GL, Henley A, RowlandTC, Sahai A, Griffin Mand BirckbichlerPJ (1996) Enhanced apoptosis in transformed human lung fibroblasts after exposure to sodium butyrate. In Vitro Cell. Dev. Biol. Anim. 32: 505-513

15. Conway RM, Madigan MC, Penfold PL and Billson IA (1995) Induction of apoptosis by sodium butyrate in the human Y-79 retinoblastoma cell line. Oncol. Res. 7: 289-297

16. Perrine SP and Faller DV (1993) Butyrate-induced reactivation of the fetal globin genes: A molecular treatment for the $\beta$-hemoglobinopathies. Experientia 49: $133-137$

17. McKnight GS, Hager L and Palmiter RD (1980) Butyrate and related inhibitors of histone deacetylation block the induction of egg white genes by steroid hormones. Cell, 22: 469-477

18. Tichonicky L, Santana-Calderon MA, Defer N, Giesen EM, Beck G and Kruh J (1981) Selective inhibition by sodium butyrate of glucocorticoid-induced tyrosine aminotransferase synthesis in hepatoma tissue-cultured cells. Eur. J. Biochem. 120: $427-433$

19. Plesko MM, Hargrove JL, Granner DK and Chalkley R (1983) Inhibition by sodium butyrate of enzyme induction by glucocorticoids and dibutyryl cyclic AMP. J. Biol. Chem. 258: 13738-13744

20. Hirose M, Sarui K and Sunagawa A (1985) Correlation between nuclear histone acetylation and casein messenger RNA induction in the mammary gland. J. Biochem. (Tokyo) 97: 781-789

21. Martel P, Houdebine LM, Teyssot B (1983) Effect of sodium butyrate on the stimulation of casein gene expression by prolactin. FEBS Lett. 154: 55-59

22. Cattini PA, Kardami $E$ and Eberhardt NL (1988) Effect of butyrate on thyroid hormone-mediated gene expression in rat pituitary tumour cells. Mol. Cell. Endocrinol. 56: 263-270

23. Weingarten $D$ and de Vellis J (1980) Selective inhibition by sodium butyrate of the glucocorticoid induction of glycerol phosphate dehydrogenase in glial cultures. Biochem. Biophys. Res. Commun. 93: 1297-1304

24. Weingarten DP, Kumar S and de Vellis J (1981) Paradoxical effects of sodium butyrate on the glucocrticoid inductions of glutamine synthetase and glycero phosphate dehydrogenase in C6 cells. FEBS Lett. 126: 289-291

25. Littlefield BA and Cidlowski JA (1984) Increased steroid responsiveness during sodium butyrate-induced 'differentation' of $\mathrm{HeLa}_{3}$ cells. Endocrinology 114: $566-575$

26. Reik A, Schütz G and Stewart AF (1991) Glucocorticoids are required for establishment and maintenance of an alteration in chromatin structure: induction leads to a reversible disruption of nucleosomes over an enhancer. EMBO J. 10 2569-2576

27. Archer TK, Lefebvre P, Wolford RG and Hager GL (1992) Transcription factor loading on the MMTV promoter: a bimodal mechanism for promoter activation. Science 255: 1573-1576

28. Bresnick EH, Bustin M, Marsaud V, Richard-Foy H and Hager GL (1992) The transcriptionally-active MMTV promoter is depleted of histone H1. Nucleic Acids Res. 20: $273-278$
29. Truss M, Chalepakis $G$ and Beato $M$ (1992) Interplay of steroid hormone receptors and transcription factors on the mouse mammary tumor virus promoter. J. Steroid Biochem. Mol. Biol. 43: 365-378

30. Espinas ML, Roux J, Ghysdael J, Pictet R and Grange T (1994) Participation of Ets transcription factors in the glucocorticoid response of the rat tyrosine aminotransferase gene. Mol. Cell. Biol. 14: 4116-4125

31. Archer TK, FryerCJ, Lee HL, Zaniewski E, Liang Tand MymrykJS (1995) Steroid hormone receptor status defines the MMTV promoter chromatin structure in vivo. J. Steroid Biochem. Mol. Biol. 53: 421-429

32. Li Q and Wrange O (1995) Accessibility of a glucocorticoid response element in a nucleosome depends on its rotational positioning. Mol. Cell Biol. 15:4375-4384

33. Truss M, Bartsch J, Schelbert A, Hache JG and Beato M (1995) Hormone induces binding of receptors and transcription factors to a rearranged nucleosome on the MMTV promoter in vivo. EMBO J. 14: 1737-1751

34. Bresnick EH, John DS, Berard DS, LeFebvre P and Hager GL (1990) Glucocorticoid receptor-dependent disruption of a specific nucleosome on the mouse mammary tumor virus promoter is prevented by sodium butyrate. Proc. Natl. Acad. Sci. USA 87: 3977-3981

35. Bartsch J, Truss M, Bode J and Beato M (1996) Moderate increase in histone acetylation activates the mouse mammary tumor virus promoter and remodels its nucleosome structure. Proc. Natl. Acad. Sci. USA 93: 10741-10746

36. Yoshida M, Kijima M, Akita Mand Beppu T (1990) Potentand specific inhibition of mammalian histone deacetylase both in vivo and in vitro by trichostatin A. J. Biol. Chem. 265: 17174-17179

37. Yoshida M, Horinouchi S and Beppu T (1995) Trichostatin A and trapoxin: novel chemical probes for the role of histone acetyation in chromatin structure and function. BioEssays 17: 423-430

38. Strasser-WozakEM, Hattmannstorfer R, Hala M, Hartmann BL, FiegI M, Geley S and Kofler R (1995) Splice site mutation in the glucocorticoid receptor gene causes resistance to glucocorticoid-induced apoptosis in a human acute leukemic cell line. Cancer Res. 55: 348-353

39. Bansal N, Houle A and Melnykovych G (1991) Apoptosis: mode of cell death induced in T leukemia lines by dexamethasone and other agents. FASEB J. 5 : 211-216

40. Geley S, Hartmann BL, Hala M, Strasser-Wozak EM, Kapelari K and Kofler, R (1996) Resistance to glucocorticoid-induced apoptosis in human T-cell acute lymphoblastic leukemia CEM-C1 cells is due to insufficient glucocorticoid receptor expression. Cancer Res. 56: 5033-5038

41. Zhou F and Thompson EB (1996) Role of c-jun induction in the glucocorticoidevoked apoptotic pathway in human leukemic lymphoblasts. Mol. Endocrinol. 10: $306-316$

42. Brunet CL, Gunby RH, Benson RSP, Hickman JA, Watson AJM and Brady G (1998) Commitment to cell death measured by loss of clonogenicity is separable from the appearance of apoptotic markers. Cell Death Differ. 5: 107-115

43. Bell PA and Jones CN (1982) Cytotoxic effects of butyrate and other 'differentiation inducers' on immature lymphoid cells. Biochem. Biophys. Res. Commun. 104: 1202-1208

44. Csordas A (1990) On the biological role of histone acetylation. Biochem. J. 265: $23-38$

45. Turner BM (1991) Histone acetylation and control of gene expression. J. Cell Sci. 99: $13-20$

46. Bradbury EM (1992) Reversible histone modifications and the chromosome cell cycle. BioEssays 14: $9-16$

47. Brownell JE and Allis CD (1996) Special HATs for special occasions: linking histone acetylation to chromatin assembly and gene activation. Curr. Opinion Gen. Dev. 6: $176-184$

48. McBain JA, Eastman A, Nobel CS and Mueller GC (1997) Apoptotic death in adenocarcinoma cell lines induced by butyrate and other histone deacetylase inhibitors. Biochem. Pharmacol. 53: 1357-1368

49. Matsuo N, Yamada K, Noda S, Yamashita K, Okuda A, Kimura G and Sugano M (1994) Reversible proliferation arrest of rat $3 Y 1$ fibroblasts and selective killing of Simian virus transformed derivation of $3 \mathrm{Y} 1$ by short-chain fatty acids. Int. J. Oncol. 5: 655-660

50. Schwartzman RA and Cidlowski A (1993) Mechanism of tissue-specific induction of interrnucleosomal deoxyribonucleic acid cleavage activity and apoptosis by glucocorticoids. Endocrinology 133: 591-599

51. Lee E, Furukubo T, Miyabe T, Yamauchi A and Kariya K (1996) Involvement of histone hyperacetylation in triggering DNA fragmentation of rat thymocytes undergoing apoptosis. FEBS Lett. 395: 183-187 
52. Nieto MA, Gonzales A, Gambon F, Diaz-Espeda F and Lopez-Rivas A (1992) Apoptosis in human thymocytes after treatment with glucocorticoids. Clin. Exp. Immunol. 88: $341-344$

53. Helmberg A, Auphan N, Caelles C and Karin M (1995) Glucocorticoid-induced apoptosis of human leukemic cells is caused by the repressive function of the glucocorticoid receptor. EMBO J. 14: 452-460

54. Morman MR and Thompson EB (1977) Characterization of a glucocorticoidsensitive human lymphoid cell line. Cancer Res. 37: 3785-3791
55. Nicoletti I, Migliorati G, Pagliacci MC, Grignanai F and Riccardi C (1991) A rapid and simple method for measuring thymocyte apoptosis by propidium iodide staining and flow cytometry. J. Immunol. Methods 139: 271-279

56. Sgonc R and Wick G (1994) Methods for the detection of apoptosis. Int. Arch. Allergy Immunol. 105: 327-332 